\section{A case report of severe atypical Weil's syndrome in a department of gastroenterology}

\author{
Yi-Qun Hu, ${ }^{1}$ Jing-Jin Song, ${ }^{2}$ Ya-Pi Lu, ${ }^{1}$ \\ Xun-Ding Lin, ${ }^{1}$ Bayasi Guleng, ${ }^{1}$ \\ Jian-Lin Ren ${ }^{1}$ \\ 1Department of Gastroenterology and \\ 2Department of Vasculocardiology, \\ Zhongshan Hospital affiliated to Xiamen \\ University, China
}

\begin{abstract}
Leptospirosis is a reemerging zoonosis of global importance. Outbreaks are related to agricultural environments and exposure to flooding, and it is primarily found in tropical countries. Although cases with typical presentation are relatively easy to diagnose and treat, atypical cases make it difficult to differentiate from other diseases, especially those patients with digestive symptoms who present to departments of gastroenterology. A 54-year old man was admitted to hospital for jaundice, vomiting and fatty liver. leptospirosis antibody test and polymerase chain reaction did not help us to make a definite diagnosis. However, based on the patient's presentation, physical characteristics and the muscular biopsy, Weil's syndrome was diagnosed. This is a reminder that leptospirosis may be found in a developed city of southern China. Clinical experience, disease characterizations, physical signs and biopsy should be applied as more efficient ways to recognize atypical cases of leptospirosis.
\end{abstract}

\section{Introduction}

Leptospirosis is a globally zoonotic disease. Early diagnosis is critical because antibiotic treatment is most effective in the initial stage, ${ }^{1}$ However, it is quite possible for patients to be misdiagnosed or diagnosed incorrectly in a department of gastroenterology for the cases with jaundice, vomiting and fatty liver at early onset. Even though culture and microscopic agglutination testing are gold standards for the diagnosis of leptospirosis, results are often negative at the initial stage. Serological tests have low sensitivity and low specificity at this phase. Polymerase chain reaction (PCR) has been demonstrated to be a valuable tool but it is not available in most developing countries. Thus, clinical experience, disease characterizations, examination of physical signs and biopsy should be used as more efficient tools to recognize and diagnose leptospirosis.

\section{Case Report}

A 54-year old man who suddenly presented with vomiting of brown stomach contents accompanied by nausea, followed by fever and asthenia, no abdominal pain and melena was admitted to the department of gastroenterology for suspected hematemesis or other digestive diseases. Vital signs were unstable and blood pressure was between $60-70 / 40-50 \mathrm{~mm} / \mathrm{Hg}$. The patient had a history of hypertension, jaundice was found in sclera and skin, bulbar conjunctiination showed moist rales on the left lung field. Results of blood tests were: WBC $11.0 \times 10^{9} / \mathrm{L}, \mathrm{N}$ 74.9 , Hb $137 \mathrm{~g} / \mathrm{L}$, PLT $94 \times 10^{9} / \mathrm{L}$, ALT $378.6 \mathrm{u} / \mathrm{L}$ of abnormal liver function, CREA $293.2 \mathrm{umol} / \mathrm{L}$ of renal function. Lung computed tomography (CT) showed pneumonia of low-lobe of both lungs (Figure 1A). Abdominal CT scan implicated a severe fatty liver (Figure 1B). Diagnosis on admission was: i) suspected infectious shock; ii) acute kidney failure; iii) suspected virus hepatitis. The patient was treated with colloid to fluid expansion, dopamine to raise blood pressure, efotaxime antibiotic, gluthion to protect liver function, and transmetil to reduce jaundice. The second day, the patient complained of headache while blood pressure was unstable and tended to be lower than before. Then patient was transferred to the intensive care unit (ICU) and general tests were performed as follows: urine bilirubin $1+$, urine occult blood 3+, AST 633.40 u/L, CK 2449.80 u/L, CKMB 70.70 u/L, HBDH $268.70 \mathrm{u} / \mathrm{L}$, LDH $398.10 \mathrm{u} / \mathrm{L}$, blood serum troponin T $410.70 \mathrm{pg} / \mathrm{mL}$, ALB $22.40 \mathrm{~g} / \mathrm{L}$, CREA $238.90 \mathrm{umol} / \mathrm{L}$, GLU $13.19 \mathrm{mmol} / \mathrm{L}$, blood clotting function of APTT-STAG $46.50 \mathrm{sec}$, D-DSTAG $2020.00 \mathrm{ng} / \mathrm{mL}$, FIB $4.58 \mathrm{~g} / \mathrm{L}$, INR-STAG 1.43, PT-Ratio 1.31, PT-STAG $16.90 \mathrm{~s}$, repeated bacterial culture negative, hs-CRP $243.00 \mathrm{mg} / \mathrm{L}$, HBsAg (-), HBsAb (+), anti-HCV (-), Widal test

\section{A}

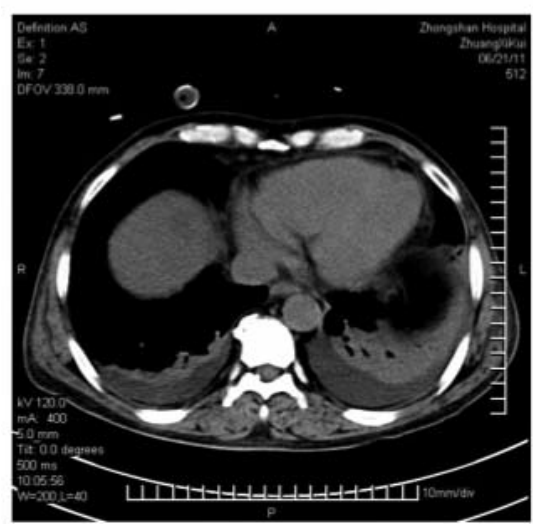
va showed slight hyperemia, and physical exam-

Correspondence: Bayasi Guleng, Department of Gastroenterology, Zhongshan Hospital affiliated to Xiamen University, 201 Hubin South Road, Xiamen, Fujian Province, 361004 China.

Tel. +86.592.2993170 - Fax: +86.592.2212328

E-mail: bayasi8@gmail.com

Key words: atypical Weil's syndrome, department of gastroenterology, developing city.

Contributions: $\mathrm{YQH}, \mathrm{BG}$, study concept; $\mathrm{BG}$, wrote the paper; YQH, JJS, YPL, XTL, collected information and data; JLR, proofread the manuscript.

Conflict of interests: the authors report no potential conflict of interests.

Funding: this study was supported by a Major Foundation of Xiamen Municipal Health Bureau (n. 3502z20077038).

Received for publication: 26 March 2012.

Revision received: 12 June 2012.

Accepted for publication: 10 July 2012.

This work is licensed under a Creative Commons Attribution NonCommercial 3.0 License (CC BYNC 3.0).

(C) Copyright Y-Q Hu et al., 2012

Licensee PAGEPress, Italy

Gastroenterology Insights 2012; 4:e18

doi:10.4081/gi.2012.e18

and Weil-Felix reaction (-), PLT $19.00 \times 10^{9} / \mathrm{L}$, tumor marker CA199 40.65 U/mL, antineutrophilic cytoplasmic antibodies of myeloperoxidase and proteinase $3(-)$, unheated serum reagm test (-), microspironema pallidum (-), anti-HIV (-), epidemic hemorrhagic fever antibody IgM (-), cytomegalo virus antibody IgM (-), Herpes Simplex Virus antibody IgM (-), Rubella virus $\operatorname{Ig} M(-)$, Toxoplasma IgM (-), antiLeptospira IgG (+) 24U/mL. Anti-Leptospira IgM

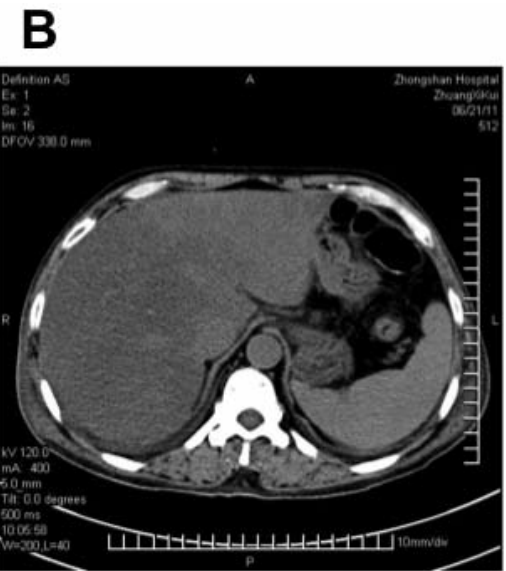

Figure 1. A) computed tomography (CT) image from the lung; B) CT image showing the severe fatty liver. 
still showed negative, anti-Leptospira IgG showed $21 \mathrm{U} / \mathrm{mL}$ at two weeks and there was no change in antibody titer. During hospitalization, the patient also complained of gradual extremity muscular soreness asthenia, a rise in local skin temperature that was characterized by muscle swelling (Figure 2A-D) and, towards the end of his stay in hospital, he found it difficult to walk. However, the anti-Leptospira $\operatorname{IgG}(+)$ and anti-Leptospira IgM (-) results did not indicate clear diagnosis of Leptospira infection, based on the symptom of headache, jaundice and renal failure, we suspected leptospirosis and performed Ultrasound B to observe the changes in leg muscles continuously. Mixed cyst and solid changes were observed between fat lining and muscular tissue of four limbs; the flow was observed in the cyst area when these were squeezed or pushed (Figures 3A-F). A biopsy was performed (Figure 3G) and fluid samples were taken from the gastronomies muscle tissue of the right leg; no parasites or worm eggs were found. Immunohistochemistry analysis showed infiltration of inflammatory cells, severe edema between connective tissue and striated muscle bundle, and dissolution and necrosis of muscle fibers (Figure 4A); these characteristics agree with those of pyomyositis. Furthermore, multi hook-like and curve shaped pathogens were detected on biopsy samples under Giemsa staining and confirmed as leptospira (Figure 4A and B). Once Leptospirosis was suspected, erythromycin macrolide antibiotics were started. Blood pressure returned to normal, swelling of the limb muscles went down and ecdysiasts appeared (Figure 2B and D). Liver and renal function was gradually restored. Subcutaneous multi-nodules in four limbs were revealed by ultrasound B and the patient gradually started to walk by himself again. The patient was discharged from hospital and follow up was initiated.

\section{Discussion}

Leptospirosis is a globally zoonotic disease that is difficult to diagnose at the early stage. It can, therefore, be misdiagnosed and nonspecific presentation in a department of gastroenterology of some cases with digestive symptom, such as jaundice, vomiting, fatty liver, etc., can also lead to an incorrect diagnosis. The early diagnosis of leptospirosis is critical since antibiotic treatment is most effective in the initial stage..$^{2-4}$ Although culture and the microscopic agglutination test are gold standard methods for its diagnosis, it is difficult to obtain a positive result at early onset. Serological tests have low sensitivity and low specificity at this stage. PCR has been shown to be a valuable diagnostic tool, but it is not available in most developing countries and in

\section{Before treat}
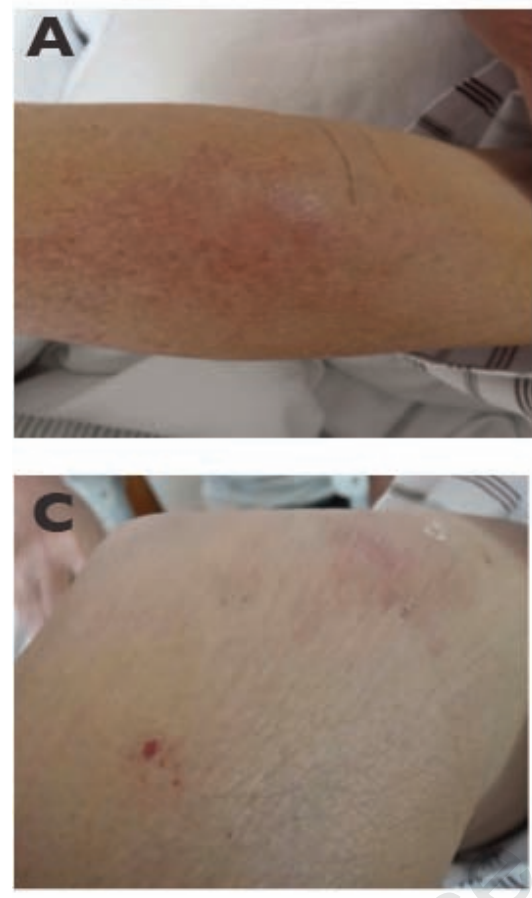

After treat
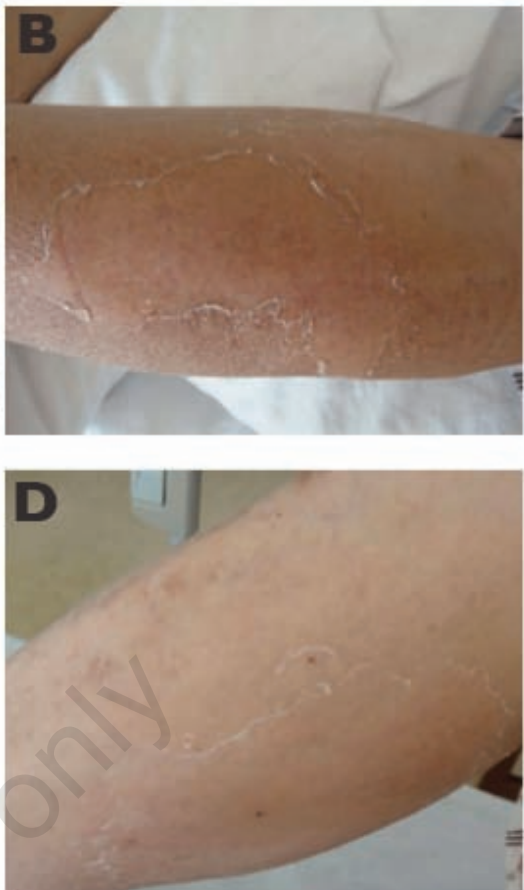

Figure 2. A,B) image of swelling right forearm; C,D) image of swelling thigh.
6011-6-30
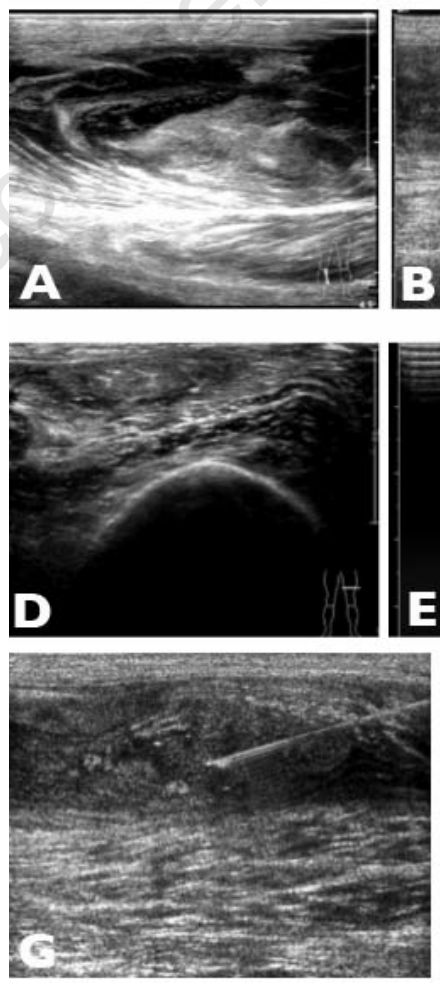

\section{1-7-8}
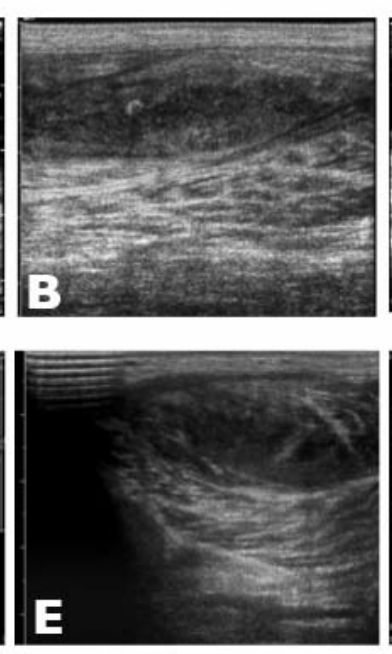

6011-7-18
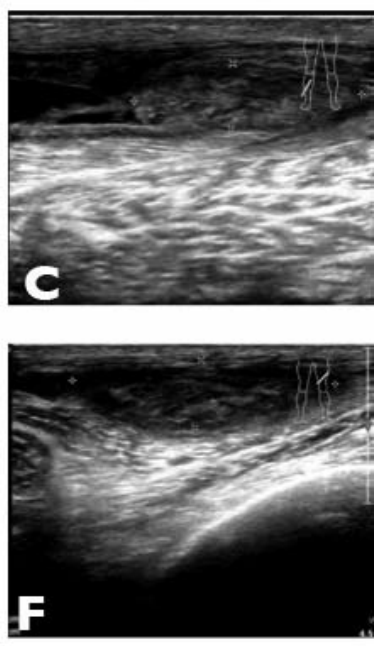

Figure 3. A,B,C) ultrasound B images of right shank gastrocnemius muscle; D,E,F) ultrasound $B$ images of left thigh quadriceps femoris muscle; G) image from right shank gastrocnemius muscle puncture. 

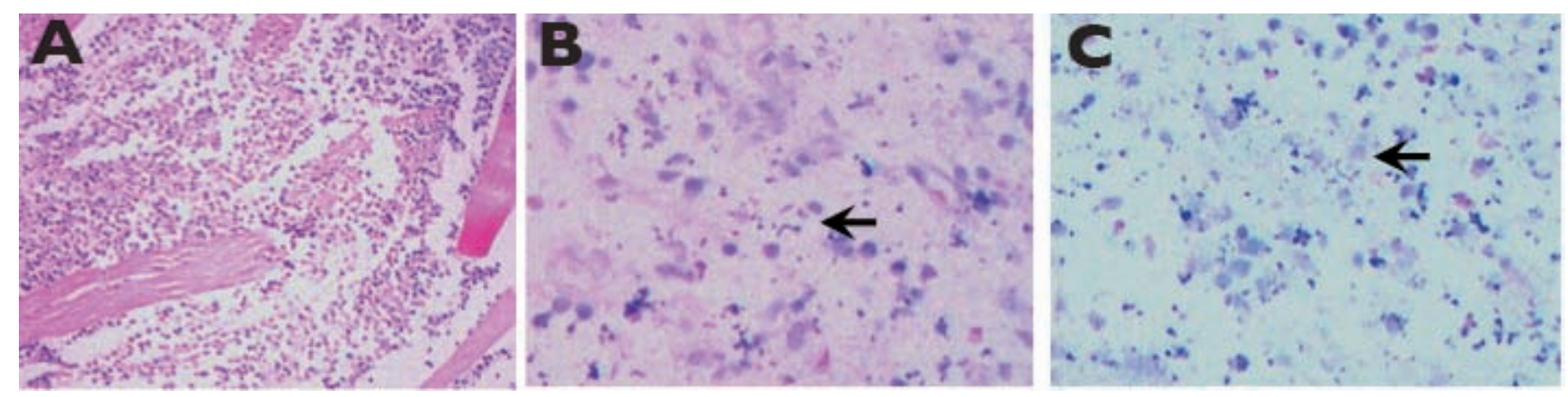

Figure $4 \mathrm{~A}$ ) Haematoxylin and Eosin staining (20x) image shows the inflammatory cells (pyomyositis) and muscular necrosis from fight thigh musculus biopsy; B), C): Giemsa Staining (100x) shows Leptospira on right thigh musculus biopsy.

some places with a low incidence of leptospirosis. Thus, clinical and diagnostic experience should be applied as a more efficient way to arrive at a final diagnosis.

In this case, leptospirosis was diagnosed mainly on the basis of the biopsy result: the finding of microorganism in the musculus tissue. The anti-IgM, anti-IgG and PCR tests did not provide clear evidence of Leptospira,,$^{5}$ the lymphocyte counts did not show any clear signs of leptospiral infection, and the symptoms of diffused myalgia, headaches, jaundice, conjunctival suffusion, renal damage and blood thrombocytopenia were presented during the disease course. In the beginning this created some confusion and macrolide antibiotics were given on the basis of a possible diagnosis of a special infection. The patient responded to the antibiotic treatment. According to documented literature and case reports, ${ }^{6} \operatorname{IgM}$ of Leptospirosis is not always positive. The fact that the patient's IgG titre did not show any step-up confused the diagnosis. There was the suspicion that the patient's immune system had been damaged at the start of this severe case, and that this had modified the immune response, resulting in undetectable levels of IgM antibody. In a small proportion of patients IgG antibody might appear first.

According to epidemic data, fewer severe forms have been recorded in recent years in southern China, especially in developed cities. On the other hand, the majority of leptospiral infection cases are either subclinical or mild in presentation. These are resolved without any complications, and only a small number of cases with resultant multiple system organ failure. ${ }^{7}$ Mortality in severe cases is still high and some data show that in older patients with acute renal failure and jaundice mortality is approximately $15-20 \% .^{8}$ Some of these cases had been misdiagnosed and treated as Hepatitis B, C or other diseases and this could be one of reasons for the high mortality. This urged us to improve our medical technologies and diagnostic ability, to acquire a better understanding of the clinical presentation of leptospirosis (especially for Weil's syndrome), and to improve the prognosis of these cases in our department of gastroenterology.

\section{Conclusions}

In recent years, the droughts and flooding caused by the global climate changes has had an impact on the incidence leptospirosis, and a marked increase in incidence rates and regional multiple outbreaks has been seen. ${ }^{9}$ However, the main risk factors for leptospirosis remain unchanged: male gender, occupational exposure, and contact with cattle, pigs or rodent. ${ }^{8,10}$ Cases of Weil's syndrome tend to be more common in a department of gastroenterology, and further studies based on clinical and pathogenic factors are required.

\section{References}

1. Desai S, Lierz M, Espelage W, et al. Resurgence of field fever in a temperate country: an epidemic of leptospirosis among seasonal strawberry harvesters in
Germany in 2007. Clin Infect Dis 2009;48:691-7.

2. Abgueguen P, Delbos V, Blanvillain J, et al. Clinical aspects and prognostic factors of leptospirosis in adults. Retrospective study in France. J Infect 2008;57:171-8.

3. Behera BCR, Pandey A, Mohan A, et al. Coinfections due to leptospira, dengue and hepatitis E: a diagnostic challenge. J Infect Dev Ctries 2009;4:48-50.

4. Michalopoulos A, Pappas G, Papadakis E, et al. Leptospirosis in a European intensive care unit. Scand J Infect Dis 2010; 42:69-71.

5. Craig SB, Graham GC, Burns MA, et al. Lymphopenia is observed regularly in the acute (leptospiraemic) phase but not the immune phase of leptospirosis. Trans $\mathbf{R}$ Soc Trop Med Hyg 2009;103:958-60.

6. Pischke S, Ehmer U, Schedel I, et al. Of guinea pigs and men: an unusual case of jaundice. Z Gastroenterol 2010;48:33-7.

7. Vijayachari P, Sugunan AP, Shriram AN. Leptospirosis: an emerging global public health problem. J Biosci 2008;33:557-69.

8. Abdulkader RC, Silva MV. The kidney in leptospirosis. Pediatr Nephrol 2008;23: 2111-20.

9. Victoriano AF, Smythe LD, GlorianiBarzaga N, et al. Leptospirosis in the Asia Pacific region. BMC Infect Dis 2009;9:147.

10. Storck CH, Postic D, Lamaury I, Perez JM. Changes in epidemiology of leptospirosis in 2003-2004, a two El Niño Southern oscillation period, Guadeloupe archipelago, French West Indies. Epidemiol Infect 2008;136:1407-15. 